This item was submitted to Loughborough's Research Repository by the author.

Items in Figshare are protected by copyright, with all rights reserved, unless otherwise indicated.

\title{
A modal-based derivation of transient pressure distribution along the tyre- road contact
}

PLEASE CITE THE PUBLISHED VERSION

http://dx.doi.org/10.4271/2009-01-0457

PUBLISHER

(C) SAE International

VERSION

AM (Accepted Manuscript)

LICENCE

CC BY-NC-ND 4.0

\section{REPOSITORY RECORD}

Tsotras, Achillefs, and George Mavros. 2013. "A Modal-based Derivation of Transient Pressure Distribution Along the Tyre-road Contact”. figshare. https://hdl.handle.net/2134/11393. 
This item was submitted to Loughborough's Institutional Repository

(https://dspace.Iboro.ac.uk/) by the author and is made available under the following conditions:

Copyright (C) 2009 SAE International. This paper is posted on this site with permission from SAE International, and is for viewing only. Further use or distribution of this paper is not permitted without permission from SAE 


\title{
A modal-based derivation of transient pressure distribution along the tyre-road contact
}

\author{
Achillefs Tsotras and George Mavros \\ Loughborough University, UK
}

Copyright (C) 2009 SAE International

\begin{abstract}
The two-dimensional, frictional tyre-road contact interaction is investigated. A transient contact algorithm is developed, consisting of an analytical belt model, a non linear sidewall structure and a discretized viscoelastic tread foundation. The relationship between the magnitude/shape of the predicted two-dimensional pressure distribution and the corresponding belt deformation is identified. The effect of vertical load and the role of sidewall non linearity are highlighted. The modal expansion/reduction method is proposed for the increase of the computational efficiency and the effect of the degree of reduction on the simulation accuracy is presented. The qualitative results are physically explained through the participation of certain modes in the equilibrium solution, offering directions for the application of the modal reduction method in shear force oriented tyre models.
\end{abstract}

\section{INTRODUCTION}

Tyres play a crucial role in a vehicle's dynamic behaviour potential, as the forces developed in the tyreroad contact area are responsible for any change in the vehicle's motion. The developed forces simultaneously act as a transient excitation of the tyre structure and a closed loop of interaction is established with the developed response affecting the tyre force potential. The tyre's contact behaviour non linearity and the frictional nature of interaction, though, impose severe difficulty in the simulation of the tyre-road interaction mechanism.

The tyre contact strain and stress field along the interface area has been the topic of extensive research, as the resulting pressure distribution directly affects the shear force potential of the tyre. Numerous methods for the experimental derivation of the contact pressure distribution have been proposed, such as pres- sure sensing contact surfaces [1, 2, 3], light absorbtion/reflection techniques [4] and ultrasonic based measurements [5]. The accurate simulation, though, of the pressure distribution development still comprises a challenging research aim for reasons associated with the tyre's shape, structural anisotropy and the viscoelastic phenomena related to the interaction.

The rapid development of the finite element methods enabled the representation of tyre contact stress and strain field with great accuracy [6, 7], but the necessary fine discretization imposes a huge computational load. If this shortcoming is overcome or neglected, the discretized modelling approach can be extended towards accurate transient tyre modelling [8]. If the transient nature of tyre contact behaviour is, though, neglected, analytical carcass deformation representations can predict the equilibrium pressure distribution. A membrane shell model, for example, was combined with a contact stiffness foundation in $[9,10]$.

The well established ring on elastic foundation model, introduced in [11], still represents the state-of-theart of two-dimensional tyre carcass modelling. Its vibrational behaviour correlation with the actual tyre structure has been experimentally verified in numerous studies [12, 13, 14]. Although an analytical, ring based, contact solution has been presented in [1], in the majority of the ring model applications, the solution is acquired through the modal expansion/reduction technique [15], offering a great computational advantage to the approach.

Ring based models have been often proposed as tyre belt representations under the steady state [16] or the transient approach [17]. For computational efficiency reasons, though, the contact pressure field is not usually calculated as part of the computation algorithm but artificially superimposed as an input to the friction calculation sub-model ([17], SWIFT model described 
in [18]). Analytical parabolic/trapezoidal shaped vertical pressure distribution is the common assumption along the contact length when the friction potential has to be calculated, as in [19]. This approach is believed to moderate the transient validity and potential of the model, in comparison to studies where the contact pressure field is incorporated in the simulation process [20].

Although the necessary modal/frequency range of study for the tyre behaviour simulation in vibration and vibration transmission studies has been long ago identified and discussed [21, 22, 23], the effect of the breadth of the range in the accuracy of the representation of the contact has not been investigated in depth. In the present study, the interaction between the belt frictional contact deformation and the predicted two-dimensional contact pressure distribution is investigated and the crucial role of the vertical load/deformation is identified. The study of this interaction is carried out with the development of a physical-ring based- tyre model which enables the qualitative study of the modal reduction effect on the distribution simulation accuracy. This is accomplished through the identification of the participation of each mode in the tyre contact response.

A fully transient two-dimensional tyre model has been developed and exploited for this investigation. The nature of the developed algorithm and the qualitative conclusions on the acceptable reduction level enable the future transformation of the modelling approach into a physical shear force generation simulation tool with acceptable computational demands. The present state of the research may offer guidelines for the application of modal expansion/reduction method in various tyre models, the transient nature of which imposes the real time calculation of the contact interaction stresses $[8,19]$. The huge computational load of such discretized approaches can be significantly reduced without major sacrifice of the prediction accuracy.

\section{MODEL DESCRIPTION}

The model is composed by three interacting sections:

- belt

- sidewall structure

- tread foundation

BELT REPRESENTATION The core of the proposed approach is the analytical ring on elastic foundation model. Although the solution is acquired

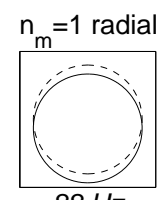

$88 \mathrm{~Hz}$

$\mathrm{n}_{\mathrm{m}}=4$ radial

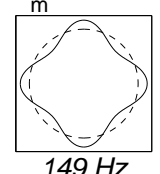

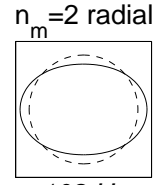

$108 \mathrm{~Hz}$
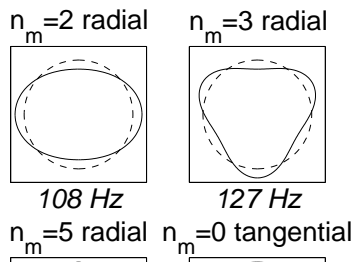

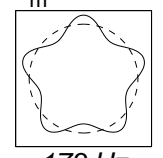

$173 \mathrm{~Hz}$

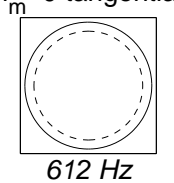

Figure 1: The mode shapes and the natural frequencies of the $n_{m}=1$ to $n_{m}=5$ radial and the $n_{m}=0$ -breathing- tangential modes.

through the modal expansion method, the simulation is accomplished in the time domain. The nature of the examined problem and the friction induced non linearity prevent the a priori association with a certain frequency range or a certain modal response and necessitates the time domain solution [24]. The usual ring inextensibility assumption is not applied here, as the proposed modal range of solution is too broad for this assumption to be valid. Additionally, the simulation of inflation, permitted only with the inclusion of an extensible belt, is vital for the solution in the non linear sidewall case. The equations of motion of the extensible ring are [15]:

$$
\begin{aligned}
& \frac{D}{R^{4}}\left(u_{r}^{\prime \prime \prime}-u_{\theta}^{\prime \prime \prime}\right)+\frac{K}{R^{2}}\left(u_{r}+u_{\theta}^{\prime}\right)+ \\
& \frac{N}{R}\left(2 u_{\theta}^{\prime}-u_{r}^{\prime \prime}\right)+k_{r} u_{r}+b d \rho \ddot{u}_{r}=q_{r}+\frac{N}{R} \\
& \frac{D}{R^{4}}\left(u_{r}^{\prime \prime \prime}-u_{\theta}^{\prime \prime}\right)-\frac{K}{R^{2}}\left(u_{r}^{\prime}-u_{\theta}^{\prime \prime}\right)- \\
& \frac{N}{R}\left(2 u_{r}^{\prime}+u_{\theta}^{\prime \prime}\right)+k_{\theta} u_{\theta}+b d \rho \ddot{u}_{\theta}=q_{\theta}
\end{aligned}
$$

$\begin{array}{ll}u_{r}(\theta) & \text { radial deformation } \\ u_{\theta}(\theta) & \text { tangential deformation } \\ b & \text { width of the ring cross section } \\ d & \text { height of the ring cross section } \\ \rho & \text { material density } \\ R & \text { ring radius } \\ D=E b d^{3} / 12 & \text { bending stiffness of the ring } \\ K=E b d & \text { membrane stiffness of the ring } \\ P_{0} & \text { inflation pressure } \\ N=P_{0} b & \text { inflation tension force } \\ k_{r} & \text { linear radial sidewall stiffness } \\ k_{\theta} & \text { linear tangential sidewall stiffness } \\ q_{r}(\theta) & \text { radial excitation } \\ q_{\theta}(\theta) & \text { tangential excitation } \\ { } & \text { angle derivative }\end{array}$

Double axis symmetry of the tyre imposes harmonic deformation functions for the deformation shapes of the modes. Each of them participates in the modal expansion twice with a $\frac{\pi}{2 n_{m}}$ angle separating the corresponding mode shapes $\left(n_{m}\right.$ being the modal number of the mode). Two modal groups 
are predicted by the expansion method. The first, low frequency, group corresponds to primarily radial deformation, while the second, high frequency, one is associated with tangential deformation. The mode shapes and the natural frequencies for some of the first modes are presented in fig. 1 .

For the radial and the tangential deformation of the jth mode it may be respectively written:

$$
\begin{aligned}
& R_{j}(\theta)=A_{j} \cos \left(n_{m}(\theta-\varphi)\right) \\
& \Theta_{j}(\theta)=B_{j} \sin \left(n_{m}(\theta-\varphi)\right)
\end{aligned}
$$

$$
\begin{array}{ll}
R_{j}(\theta), \Theta_{j}(\theta) & \text { mode shape functions } \\
A_{j}, B_{j} & \text { mode shape amplitudes } \\
\varphi & \text { excitation defined orientation angle }
\end{array}
$$

Each mode participates in the response through its modal participation factor, the time differential equation of which reads:

$$
\begin{array}{r}
\ddot{\eta}_{j}(t)+\frac{\lambda_{j}}{\rho \cdot h} \cdot \dot{\eta}_{j}(t)+\omega_{j}^{2} \cdot \eta_{j}(t)= \\
F_{j}^{\text {modal }}(t), \\
1 \leq j \leq m
\end{array}
$$

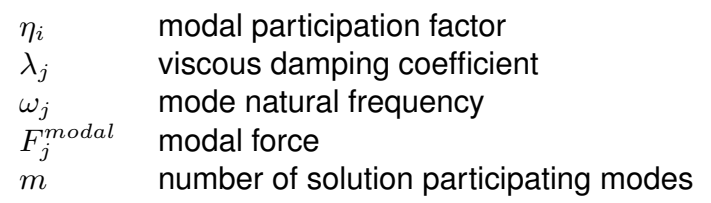

Although the belt modes are analytically derived, a constant space discretization process is applied to the ring. This process coincides with the discretization of the sidewall and tread foundations. In other words every tread and sidewall element correspond to a ring node. This permits the expression of the excitation function as a vector of constant dimension, which corresponds to the number of degrees of freedom:

$\mathbf{F}_{2 n \times 1}(t)=\mathbf{F}_{\text {inflation }}(t)+\mathbf{F}_{\text {contact }}(t)\left\{+\mathbf{F}_{\text {sidewall }}(t)\right\}$

where $n$ is the total number of nodes. Each node is associated with one radial and one tangential degree of freedom (horizontal/vertical in the global system of axis).

Under the linear sidewall assumption the sidewall effect is incorporated in the ring equations of motion (eqn.1a and 1b) and the sidewall corresponding term is not included in eqn.4. Under the non linear sidewall assumption, though, the expansion method is applied on a ring without sidewall foundation and the corresponding effect is incorporated through the force term.

The solution transformation between the modal and the space domain is achieved through the constant eigenvectors matrix $\boldsymbol{\Phi}_{2 n \times m}$. The modal mass of the

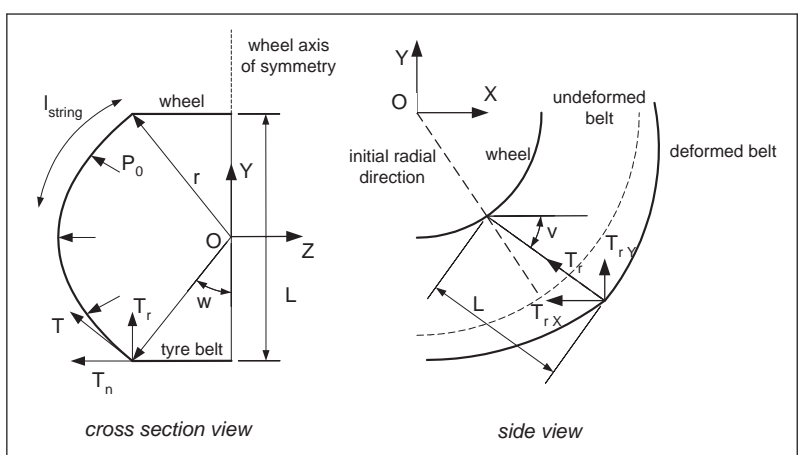

Figure 2: The radial string geometry and the resulting sidewall forces.

jth mode is defined as:

$$
M_{j}=\rho \cdot d \cdot R \cdot b \cdot \sum_{i=1}^{2 n} \Phi_{i j}^{2}
$$

The modal force of each mode can be calculated as:

$$
\begin{gathered}
\mathbf{F}_{\text {modal }}^{\text {mod }}(t)=\boldsymbol{\Phi}^{T} \times \mathbf{F}^{\text {space }}(t) \quad \Rightarrow \\
F_{j}^{\text {modal }}(t)=\frac{1}{M_{j}} F_{j}^{\text {modal }}(t)
\end{gathered}
$$

Finally, the deformation and velocity responses in the space domain are calculated from the modal participation vector according to the equations:

$$
\mathbf{U}(t)=\boldsymbol{\Phi} \times\left[\begin{array}{c}
\eta_{1}(t) \\
\vdots \\
\eta_{m}(t)
\end{array}\right], \quad \dot{\mathbf{U}}(t)=\boldsymbol{\Phi} \times\left[\begin{array}{c}
\dot{\eta}_{1}(t) \\
\vdots \\
\dot{\eta}_{m}(t)
\end{array}\right]
$$

SIDEWALL REPRESENTATION Although the ring equations of motion incorporate a two-dimensional linear stiffness foundation $\left(k_{r}, k_{\theta}\right)$, representing the sidewall of the tyre, this approach is known to result in contact reaction force overestimation [11, 23, 25, 26]. The radial buckling-softening- behaviour of the actual sidewall offers a force reduction mechanism. This behaviour is commonly modelled by a string under tension foundation, an approach also followed here, under the inextensible discretized assumption.

The tension force of an inextensible string is given by:

$$
T=P_{0} \cdot r
$$

while the geometry presented in fig. 2 proposes:

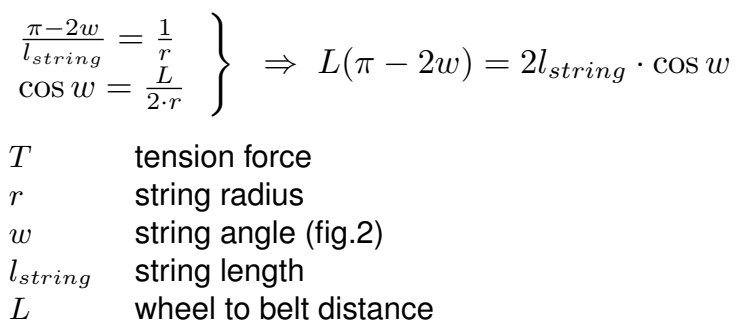




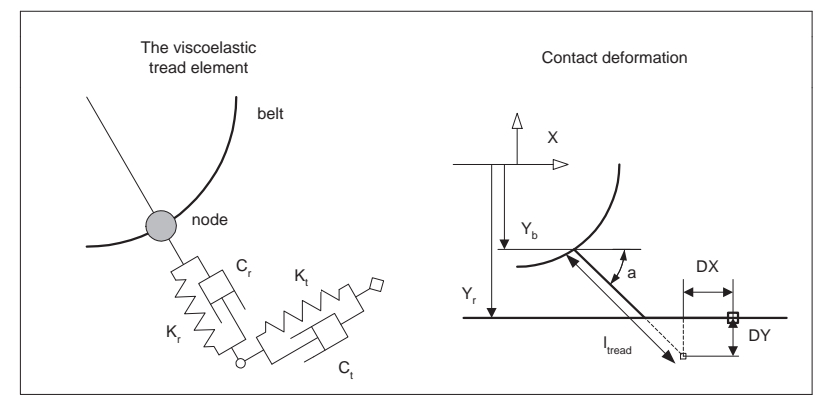

Figure 3: The tread element and its contact deformation.

The non linear eqn.9 is solved by an iteration procedure in every calculation step, using as inputs the relative wheel to road displacement and the deformation of the belt. The resulting string forces are presented in fig. 2 and their values are calculated as:

$$
\begin{aligned}
& T_{r X}=-P_{0}^{*} \cdot l_{\text {string }} \frac{\sin w}{\pi-2 w} \cos v \\
& T_{r Y}=P_{0}^{*} \cdot l_{\text {string }} \frac{\sin w}{\pi-2 w} \sin v
\end{aligned}
$$

The string sidewall behaviour is decoupled from the discretization effect by the normalization of the inflation pressure value:

$$
P_{0}^{*}=P_{0} \frac{2 \cdot \pi \cdot R}{n}
$$

The tangential sidewall behaviour is assumed to remain linear, so the corresponding stiffness is in any case incorporated in the tangential equation of motion (eqn.1b).

TREAD REPRESENTATION The tread modelling approach is a viscoelastic (Maxwell-Voigt) extension of the winkler contact stiffness theory, established in numerous studies, as a stand alone tyre model [18], or in combination with a separate carcass representation $[16,13,20]$. The tread elements are attached to the belt nodes initially with radial orientation. Slip motion potential is included in the model through the incorporation of an additional viscoelastic element and a corresponding degree of freedom. The second tread element is dimensionless under no strain condition and is attached to the free end of the radial element. Its deformation direction is vertical to the first one, while the road contact is realized at the free end of this element.

The tread viscoelastic properties are represented by the stiffness and damping matrices:

$$
\mathbf{K}=\left[\begin{array}{cc}
-K_{r} & 0 \\
0 & -K_{t}
\end{array}\right], \mathbf{C}=\left[\begin{array}{cc}
-C_{r} & 0 \\
0 & -C_{t}
\end{array}\right]
$$

$K_{r} \quad$ radial stiffness

$K_{t} \quad$ tangential stiffness

$C_{r} \quad$ radial viscous damping

$C_{t} \quad$ tangential viscous damping

The contact reaction forces, in global terms, can be calculated according to:

$\left[\begin{array}{l}F_{C X} \\ F_{C Y}\end{array}\right]=\mathbf{T}^{T}(\alpha) \mathbf{K} \mathbf{T}(\alpha)\left[\begin{array}{c}D X \\ D Y\end{array}\right]+\mathbf{T}^{T}(\alpha) \mathbf{C} \mathbf{T}(\alpha)\left[\begin{array}{c}D \dot{X} \\ D \dot{Y}\end{array}\right]$

where $\mathbf{T}$ is the angular transformation matrix:

$$
\mathbf{T}(\alpha)=\left[\begin{array}{cc}
\cos \alpha & \sin \alpha \\
-\sin \alpha & \cos \alpha
\end{array}\right]
$$

The tread global orientation angle $\alpha$ is the sum of the belt node rotation around the wheel centre, imposed by the corresponding tangential deformation and the torsional local belt deformation-expressed by the slope of the corresponding radial deformation.

$$
\alpha=u_{\theta}+\frac{\partial u_{r}}{\partial \theta}
$$

The total vertical deformation and deformation velocity of the combined element is kinematically defined by the relative road-wheel distance and the belt deformation:

$$
\begin{aligned}
& D Y=Y_{\text {road }}-\left(Y_{b}+l_{\text {tread }} \sin \alpha\right) \\
& D \dot{Y}=\dot{Y}_{r}-\dot{Y}_{b}
\end{aligned}
$$

$$
\begin{array}{ll}
D Y & \text { vertical tread deformation } \\
Y_{\text {road }} & \text { road vertical profile } \\
Y_{b} & \text { belt node vertical position } \\
l_{t} \text { read } & \text { initial radial length }
\end{array}
$$

Horizontal slip motion is controlled by the differential equation of the horizontal degree of freedom, which, under the massless assumption, transforms into a simple force equilibrium equation:

$$
\begin{array}{ll}
\quad \Sigma F_{X}=m_{t} & D \ddot{X} \Rightarrow F_{\text {tread }}+F_{\text {friction }}=0 \\
F_{\text {tread }}=-F_{C X} & \text { tread viscoelastic force } \\
F_{\text {friction }} & \text { friction force } \\
D X & \text { horizontal deformation } \\
D \dot{X} & \text { horizontal def. velocity }
\end{array}
$$

The integration of the relative slip velocity value enables the calculation of horizontal deformation:

$$
D X=\int D \dot{X} \mathrm{~d} t
$$

A number of friction laws can be incorporated in the model. The classic Coulomb one (described e.g. in [27]) is adopted here for simplicity reasons.

TRANSIENT ALGORITHM The belt, sidewall and tread sub-models are combined in a common transient algorithm. The flowchart of every discrete computation time step can be summarized as: 
1. The global position of the out-of-contact tread elements is calculated, as a function of the wheel to road relative displacement, the belt response and the tread angular orientation and deformation. Possible contact is examined through the comparison of the global tread position with the road under the no-penetration requirement.

2. The vertical deformation and deformation velocity is calculated for all the in-contact elements (eqn.14). The friction law is applied to the horizontal degree of freedom. Slip and slip velocity are calculated, as is the contact reaction excitation to the belt (eqn.15 and 16).

3. The criterion of conservation of contact $F_{C Y}>0$ is examined for the in-contact elements. The possible non-fulfillment of it results in the termination of contact.

4. The first order, initial condition, differential equation is solved for the out-of-contact elements with sufficient remaining deformation.

5. The total belt excitation vector is synthesized (eqn.4). The corresponding modal force for all the participating modes is calculated (eqn.6). The time differential equations of the modal participation factors (eqn. 3) are solved with a numerical method, while the transformation from modal to space domain (eqn.7) offers the belt response that will be used for the next time step calculation.

The response to the inflation step excitation is initially defined, prior to the contact simulation. The inflation corresponds to the breathing-0th tangential mode-participation factor. The process results in insignificant radius increase, it is, though, of great importance in the non linear sidewall case, as it offers the inflation equilibrium string geometry. Sufficient time is also granted after the contact simulation, for the establishment of equilibrium. The values of the model properties assumed for the numerical simulation are given in the Appendix.

\section{THE COMPLETE MODEL AND ITS COMPUTA- TIONAL LIMITATION}

If the theoretical calculation potential of a proposed space discretization is to be fully exploited, the required modal range can be identified by the simple observation that a wave length of a mode shape can be described by a sequence of 5 nodes (fig.4), with the last of them being shared with the following wave. The expression of this observation through the arc corre-

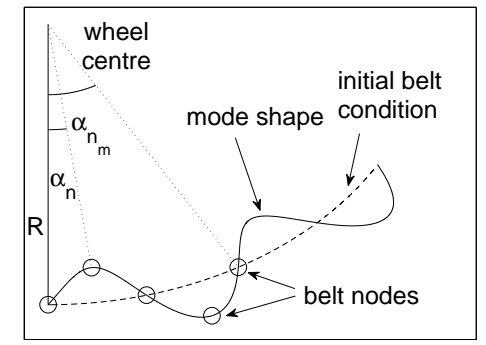

Figure 4: The relationship between the space discretization and the modal range in a complete model.

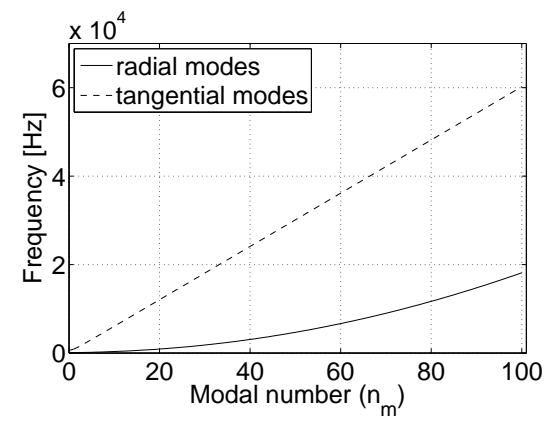

Figure 5: The total frequency range of the complete model, for $n=400$ nodes space discretization.

sponding angles reads:

$$
\left.\begin{array}{c}
\alpha_{n_{m}}=\frac{2 \pi}{n_{m}} \\
\alpha_{n}=\frac{2 \pi}{n}
\end{array}\right\} \stackrel{\alpha_{n_{m}}=4 \cdot \alpha_{n}}{\longrightarrow} n_{m}=\frac{n}{4}
$$

$$
\begin{array}{ll}
\alpha_{n_{m}} & \text { modal wavelength angle } \\
\alpha_{n} & \text { discretization angle } \\
n_{m} & \text { modal number } \\
n & \text { number of nodes }
\end{array}
$$

A belt space discretization of 400 nodes (a satisfactory coarseness for the description of the contact area development) results in a modal range $n_{m}=$ $[0, \ldots 100]$ and a total mode number $m=402$. The corresponding frequency range is presented in fig.5. The emerging huge computational load is not only associated with the number of modes, as each of them corresponds to a column of the matrix $\Phi$, but also with the assumed frequency range, imposing an extremely small time step for the simulation process.

The distribution of the damping property (assumed parallel to the stiffness one) results in the increase of the damping ratio with the modal number. In result, all the modes above a critical frequency limit demonstrate overcritical behaviour which makes their participation in the transient response insignificant for reasons associated with the energy content of their corresponding deformation. The association of the modal number of each mode with its solution partici- 


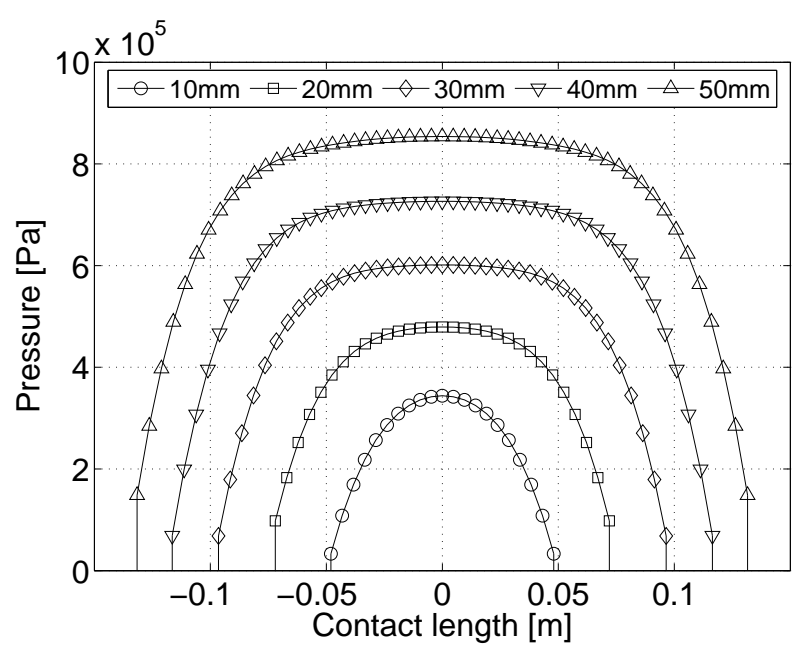

Figure 6: The vertical pressure distribution development with deformation, under the linear sidewall assumption.

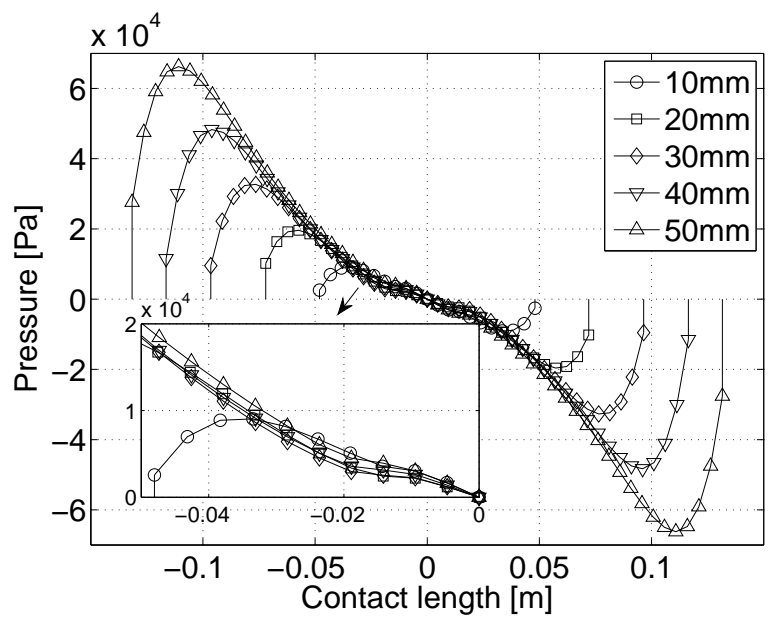

Figure 7: The horizontal pressure distribution development with deformation, under the linear sidewall assumption.

pation will be later discussed and will offer a physical explanation of the modal reduction effect on the calculation accuracy, which is the main contribution of this study.

\section{THE CONTACT BEHAVIOUR OF THE LINEAR SIDEWALL MODEL}

Prior to analyzing the tyre contact behaviour through the corresponding modal participation, it is vital to identify the effect of deformation level, as the nonlinear contact geometry affects the interaction. The vertical and tangential pressure distributions along the contact length, predicted by the linear sidewall model, are presented in figures 6 and 7 respectively, for a range of vertical displacements $d=10 \mathrm{~mm}$ to

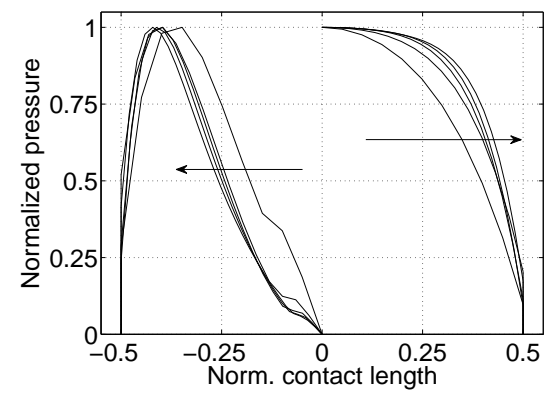

Figure 8: The effect of the deformation level on the distribution shape, through the normalized pressure values. The arrows indicate the deformation increase.

$50 \mathrm{~mm}$. The typical parabolic shape of distribution for small deformation level which transforms into trapezoidal as the deformation increases has been experimentally verified in multiple studies [28, 1, 2, 4, 29].

The horizontal pressure distribution has a typical inwards direction, mainly attributed to the frictional resistance to the outwards belt deformation, caused by the conformation of the initially circular shape to the plane road surface. It's magnitude is insignificant in the central area of the contact length, increases towards the edges but rapidly deteriorates close to the contact ends. Further discussion on the horizontal pressure distribution development can be found in $[30,28]$.

In order to highlight the effect of deformation level on the distribution shape the effect of magnitude is removed through the normalization of pressures with respect to the maximum value along the contact. The resulting pressure distributions are presented in fig.8. The transition from parabolic to trapezoidal shape with increasing deformation is evident. No shape transformation emerges in the horizontal pressure distribution, the maximum value of which moves towards the edge of the contact length with the deformation increase.

The identified distribution is associated with a corresponding variation in the belt contact deformation. The reflection of the distribution and its shape development on the equilibrium modal participation factors is sought. The radial and tangential participation factors -in absolute terms- are presented in fig. 9 for the whole deformation range.

Contact deformation is associated primarily with radial modes excitation , since the tangential participation factors are of much lower magnitude. The $n_{m}=1$ and $n_{m}=2$ (fig. 1 ) radial modes are mainly excited by the contact and the deformation increase results in 


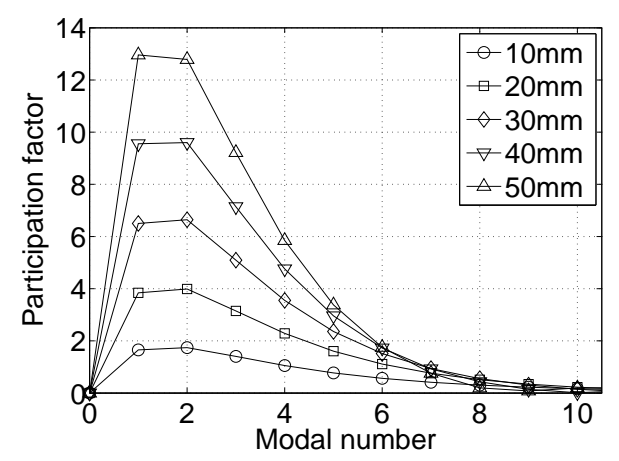

(a) Radial modes

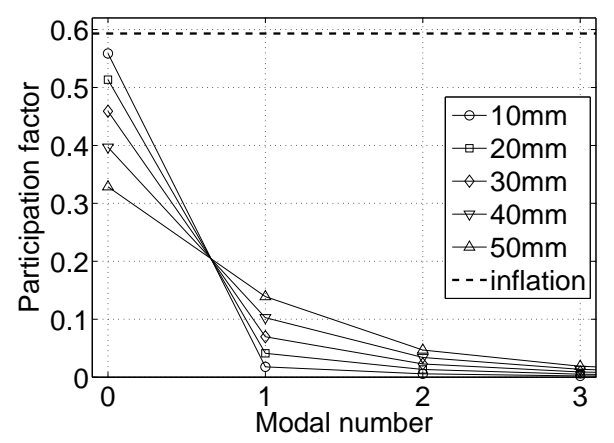

(b) Tangential modes

Figure 9: The radial and tangential modes participation factors for every load/deformation case.

a corresponding increase of their participation. For low deformation level, maximum participation corresponds to the $n_{m}=2$ mode but for high deformation participation maximizes for the $n_{m}=1$ mode. In all deformation cases radial participation factors decrease with the modal number, converging to zero value.

Maximum tangential mode participation factor corresponds to the $n_{m}=0$ mode (fig. 1 ), and is associated with the inflation process. The inflation only corresponding participation is also presented for comparison. Interestingly, the $n_{m}=0$ mode participation decreases, as the deformation process is associated with a radius decrease. The factors converge to insignificant values faster than the radial modes. Evidently, a critical modal number exists for both modal groups, above which, participation in the final solution can be neglected. This critical limit is lower for the tangential group. In other words a shorter tangential modal range can be used for the solution.

A better insight in the effect of deformation on the modal participation factors is attempted through the presentation of the normalized -to the maximum value- participation in fig.10. Interestingly, lower deformation is associated with the participation of a broader modal range. The relative participation val-

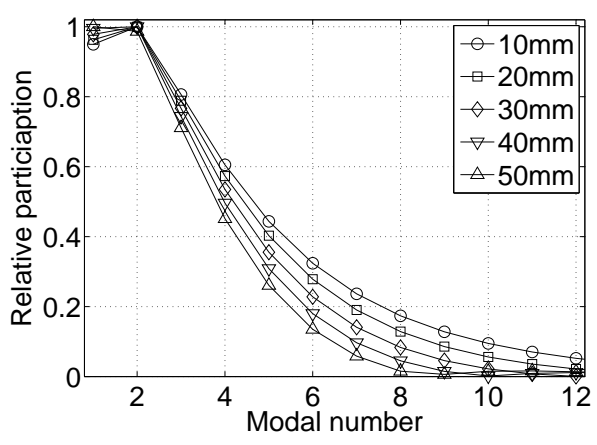

(a) Radial modes

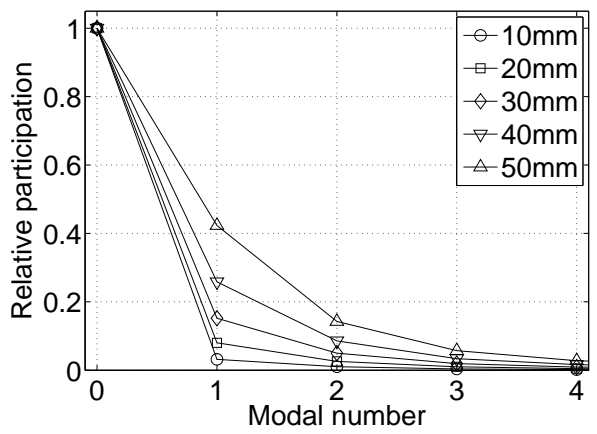

(b) Tangential modes

Figure 10: The modal participation factors, normalized to the maximum predicted value for every deformation case.

ues decrease more rapidly with the modal number, in the higher deformation cases (fig.10(a)). Higher tangential modes, in contrast, participate more in the equilibrium solution for increasing deformation level (fig.10(b)). It is evident that a broader modal range should be incorporated in the modally reduced model for the study of low load/deformation tyre operating conditions.

An explanation of this modal participation behaviour is attempted through the shape correlation between the belt contact deformation and the mode shapes. This is accomplished in fig. 11 for the $d=10 \mathrm{~mm}$ and $d=50 \mathrm{~mm}$ deformation cases, where the mode shapes of the first ten radial modes are also plotted. Their deformation magnitude has been normalized to the corresponding belt deformation for easier shape comparison. The belt deformation pattern along the contact length is better represented by the $n_{m}=5$ mode shape in the low load case, while the $n_{m}=3$ mode shape correlates better with the belt pattern in the high load case, a direct result of the contact shape nonlinearity and the relative contact length range development.

The shape correlation with a higher modal number mode shape in the low deformation case can offer an explanation to the relatively higher modal range iden- 


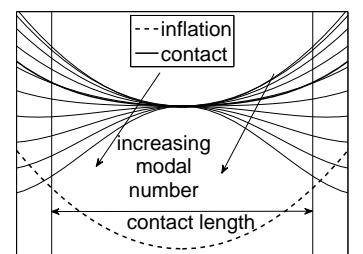

(a) $d=10 \mathrm{~mm}$

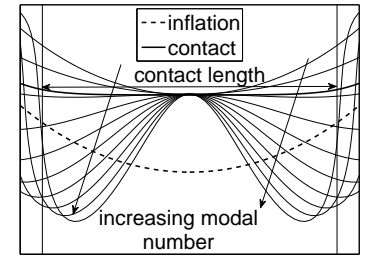

(b) $d=50 \mathrm{~mm}$
Figure 11: The shape comparison of the contact induced belt deformation along the contact length with the $n_{m}=1$ to $n_{m}=10$ radial mode shapes.

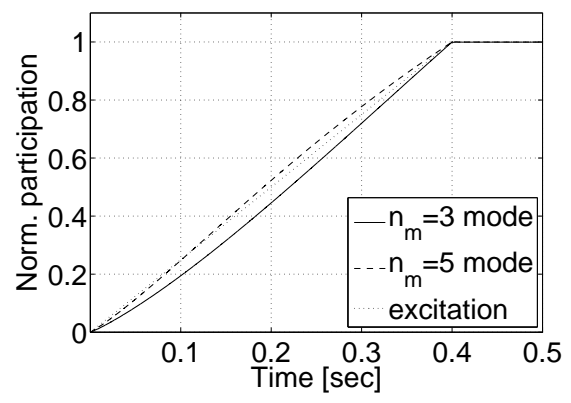

Figure 12: The normalized to their maximum absolute values participation history of the $n_{m}=3$ and $n_{m}=5$ radial modes.

tified in the relative participation factors presented in fig.10(a). Additionally, although the belt deformation pattern correlates tightly with a certain mode shape, the correspondingly high participation factors of the nearby modes reveals their importance in the total shape representation. In other words, although the contact length deformation is clearly associated with a certain mode shape, a reduced contact model cannot be generated only by this mode.

The above conclusions are evident in the time history of the $n_{m}=3$ and $n_{m}=5$ participation factors, presented in a normalized-to their maximum values-manner in fig.12. The history of the normalized deformation is also plotted for easier comparison of the mode development. The 5 th radial mode follows closely the deformation development in the early stage of the deformation, while the development of the $3 r d$ one shows an initially lagging behaviour.

\section{THE EFFECT OF THE RADIAL SIDEWALL NON- LINEARITY ON THE CONTACT BEHAVIOUR}

The incorporation of the non linear sidewall foundation, affects not only the total reaction force magnitude but also the belt deformation shape and the resulting pressure distribution. The vertical pressure distributions under the non linear sidewall assumption are presented in fig. 13 for the whole deformation range. The generation of a central concave formation is evident, the extent and magnitude of which increase with the deformation level. This pressure shape is of course accompanied by a corresponding belt deformation, which is presented in fig. 14 in comparison to the linear sidewall model predicted deformation. The effect has been analytically and experimentally studied in [1]. The transformation from parabolic/trapezoidal vertical distribution shape to the concave one is better represented by the normalized pressure values in fig.15. No significant variation emerges in the shape of the horizontal pressure distribution shape, relatively to the linear sidewall case.

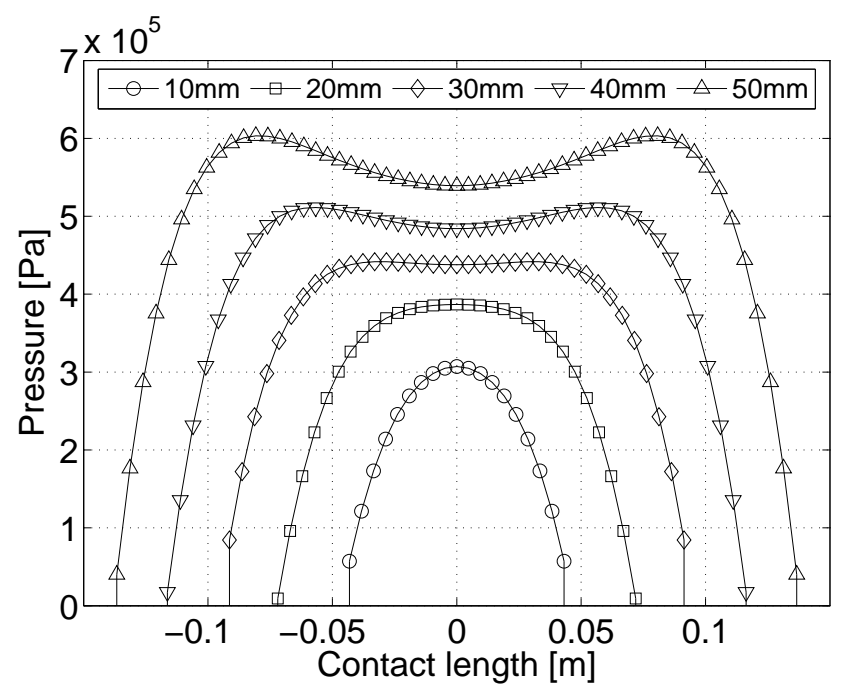

Figure 13: The vertical pressure distribution development with deformation, under the non linear sidewall assumption.

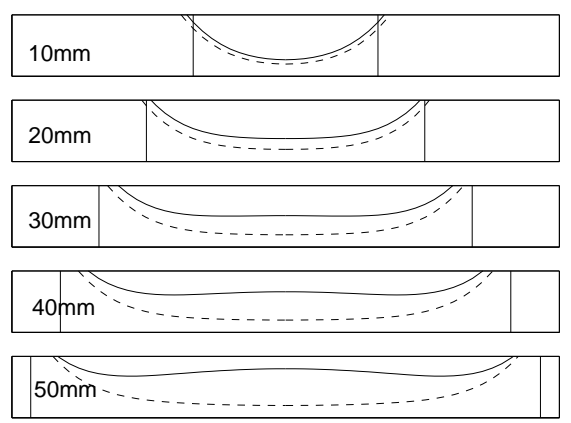

Figure 14: The belt deformation along the contact under the linear and the non linear sidewall assumptions for the whole deformation range.

The obvious question whether the non linear sidewall effect can be identified through the modal participation factors is answered by the presentation of the participation factors ratio (fig.16) to the corresponding linear sidewall ones. Three deformation levels are 


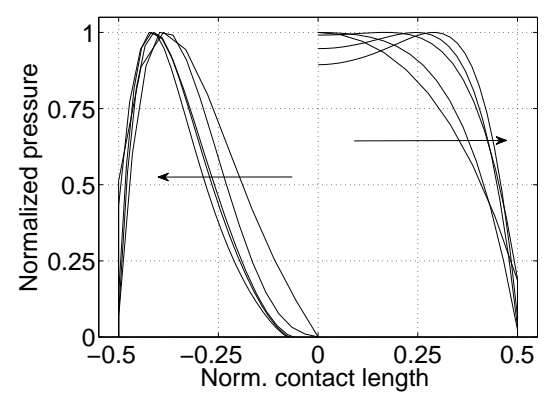

Figure 15: The effect of deformation on the distribution shape, through the normalized pressure values under the non linear sidewall assumption. The arrows indicate the deformation level increase.

examined, $d=10,30$ and $50 \mathrm{~mm}$, while for the calculation of the ratios the linear model has also been modally expanded excluding the radial sidewall foundation, the effect of which is included through the equivalent force term (eqn.6).

The buckling/softening effect of the non linear sidewall model permits the larger belt vertical deformation (evident in all cases of fig.14). This radial compliance corresponds to higher participation of the modes with modal numbers from $n_{m}=1 n_{m}=6$ evident in fig.16(c). The following magnitude anomaly in the participation ratios corresponds to the generation of the central concave deformation pattern and the accompanying shape effects in the surrounding area. Further similar areas with anomalies are repeated at higher modal numbers.

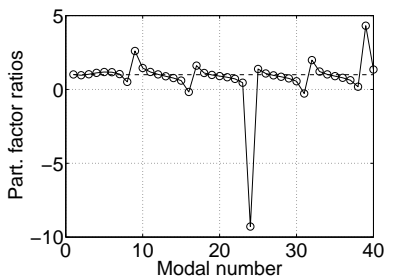

(a) $d=50 \mathrm{~mm}$

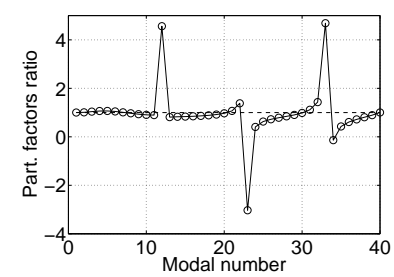

(b) $d=30 \mathrm{~mm}$

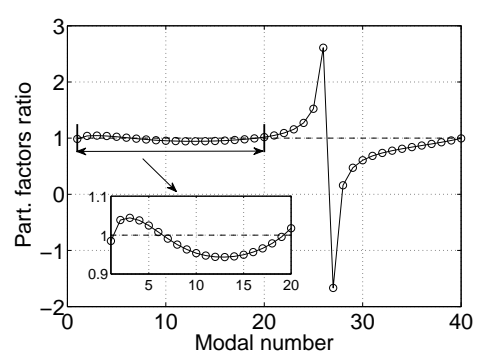

(c) $d=10 \mathrm{~mm}$

Figure 16: The ring-only participation ratios of the non linear sidewall model to the linear one.

Interestingly, the effect is associated with higher

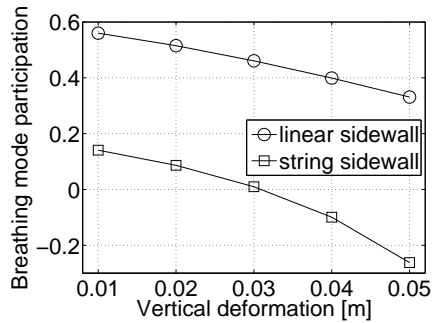

Figure 17: The breathing mode participation for the total deformation range under the linear and the non linear sidewall assumptions.

modal number mode shapes in the low deformation case. The first ratio anomaly area, is identified in the $m_{n}=26$ mode $(1401 \mathrm{HZ})$ in the $d=10 \mathrm{~mm}$ case. Extension of the concave formation in a larger area around the contact centre results in the involvement of lower modal number mode shapes. The first peak ratio value in the $d=30 \mathrm{~mm}$ case corresponds to the $m_{n}=12(570 \mathrm{~Hz})$ mode, while in the $d=50 \mathrm{~mm}$ case the $n_{m}=9(239 \mathrm{~Hz})$ mode is affected. It is evident that the accurate representation of the non linear sidewall induced belt contact behaviour requires a broader modal range in the low deformation cases where, though, the concave nature of the pressure distribution is less apparent.

The evolution of the breathing mode participation factor is presented in fig.17. The linear sidewall model predicts higher breathing mode participation in the inflation equilibrium compared to the non linear one, a direct effect of the string stiffness increase with radius. For the non-linear model, the participation of the breathing mode is higher for the same deformation range, as the buckling effect permits the higher radial deformation of the ring. Interestingly, the breathing factor becomes negative above a deformation level, a value that corresponds to radius decrease effect.

\section{THE EFFECT OF MODAL REDUCTION}

Having identified the interaction between the contact pressure distribution and the belt deformation, expressed in terms of modal participation, the effect of modal reduction on the prediction accuracy can be investigated. The effect of the modal range participating in the solution on the accuracy of prediction of the total vertical load will firstly be presented, while the resulting pressure distributions will be discussed later.

The total vertical load is presented in fig. 18 as a function of the induced deformation, for various frequency ranges. The incorporated radial and tangential modes in every reduction case are given in table 1 . The 


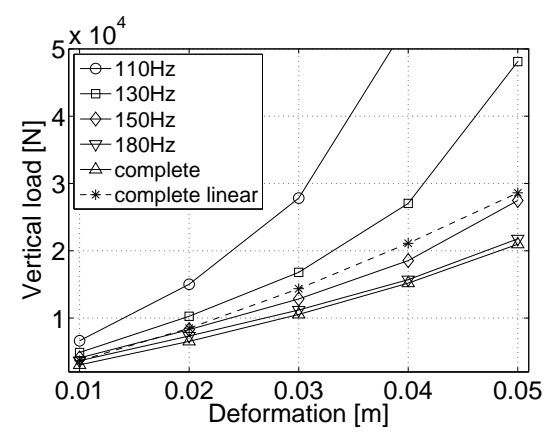

Figure 18: The vertical load predicted for the range of deformations for increasing frequency ranges of the solution.

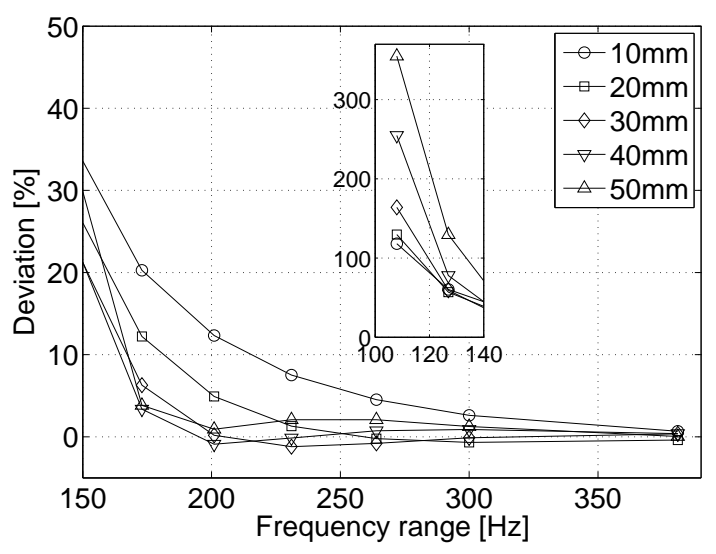

Figure 19: Percentage deviation from the complete model of the predicted vertical load as a function of the frequency range of the solution for the whole deformation range.

shorter the frequency range, the stiffer contact behaviour is predicted by the model. The deviation from the complete model increases with the deformation level. The contact behaviour for a frequency range narrower than $150 \mathrm{~Hz}$ is even stiffer than the one of the linear sidewall model (also presented in fig.18 for comparison). In other words, the stiffening effect of the incorporation of only the first three radial and the breathing modes cancels out the buckling sidewall effect. A frequency range up to $180 \mathrm{~Hz}$ (participation of 5 first radial and the breathing modes) seems to offer a good prediction accuracy for the whole deformation range.

The percentage deviation from the load prediction of the complete, non-reduced solution, is presented in fig. 19. Although an extremely narrow frequency range leads to load overestimation in the high deformation case, $350 \%$ in the $50 \mathrm{~mm}$ case compared to $120 \%$ in the $10 \mathrm{~mm}$ one for the participation of only 2 radial modes, this deviation decreases rapidly. At a frequency limit between $150 \mathrm{~Hz}$ and $300 \mathrm{~Hz}$, a larger
Table 1: Modal-frequency range correspondence

\begin{tabular}{ccc}
\hline \hline $\begin{array}{c}\text { Frequency } \\
\text { range }[\mathrm{Hz}]\end{array}$ & $\begin{array}{c}\text { Radial } \\
\text { modes }\end{array}$ & $\begin{array}{c}\text { Tangential } \\
\text { modes }\end{array}$ \\
\hline 110 & 2 & 0 \\
130 & 3 & 0 \\
150 & 4 & 0 \\
180 & 5 & 0 \\
400 & 11 & 0 \\
600 & 15 & 0 \\
800 & 18 & 1 \\
1000 & 21 & 2 \\
\hline
\end{tabular}

deviation is predicted for the $d=10 \mathrm{~mm}$ deflection compared to that of $d=50 \mathrm{~mm}$. It is obvious that the broader modal participation range associated with the low deformation level(fig.10(a)), results in higher prediction error when a common modal range is assumed in the solution of all deformation cases.

The vertical pressure distribution predicted under certain reduction assumptions is presented in fig. 20 for the $d=10$ and $50 \mathrm{~mm}$ cases. Observation reveals that achieving accuracy in the prediction of the actual

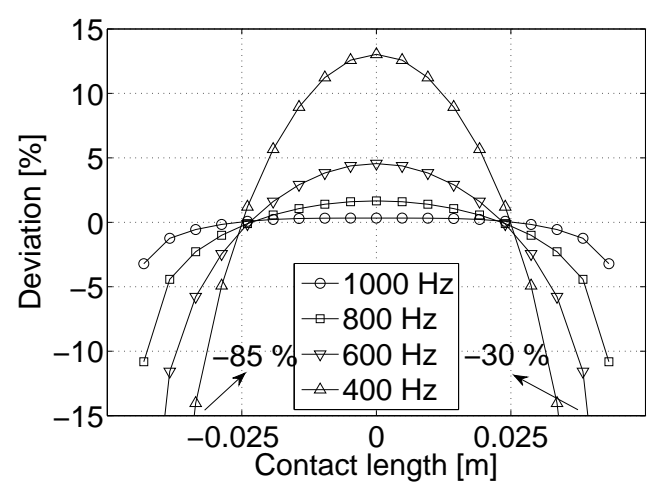

(a) $d=10 \mathrm{~mm}$

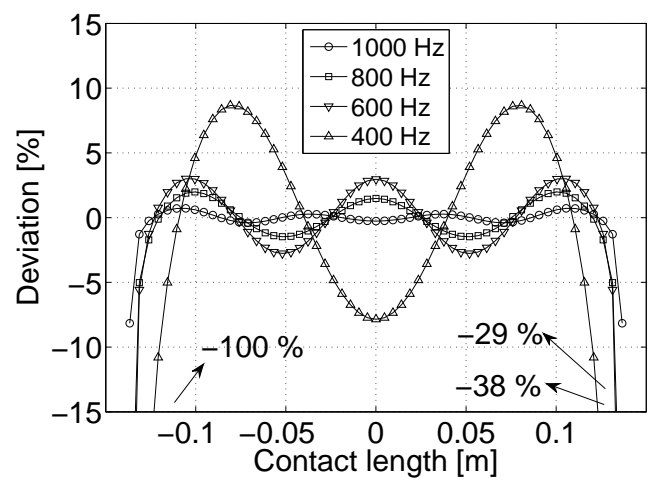

(b) $d=50 \mathrm{~mm}$

Figure 20: Vertical pressure deviation from the complete model along the contact for various levels of reduction. 


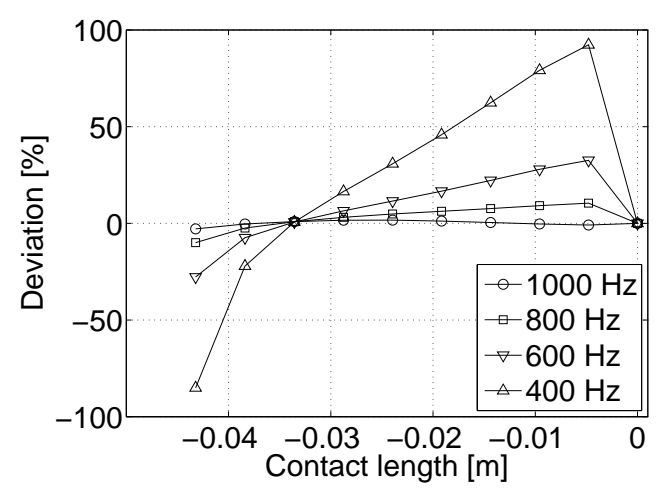

(a) $d=10 \mathrm{~mm}$

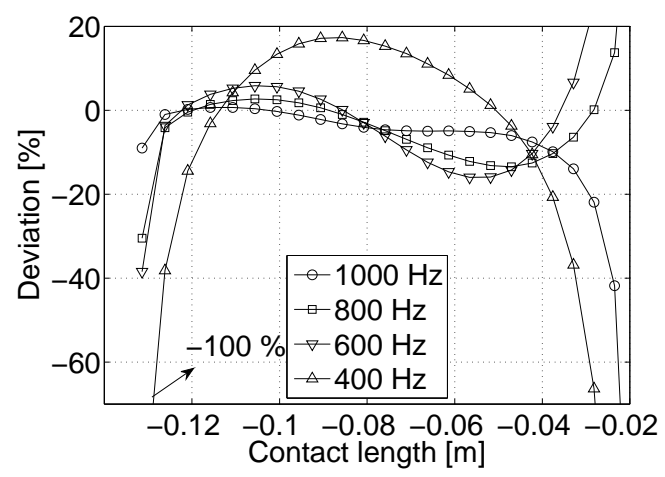

(b) $d=50 \mathrm{~mm}$

Figure 21: Horizontal pressure deviation from the complete model along the contact for various levels of reduction.

shape of the distribution requires a broader frequency range than that required for the accurate caculation of the total vertical load. In particular, $5 \%$ deviation in the total load prediction is accomplished with a modal range extending to $250 \mathrm{~Hz}$, while a similar accuracy level in the distribution requires a modal range up to $1000 \mathrm{~Hz}$. Larger deviation is noted near the edges of the contact in both examined cases, compared to the deviation level in the central contact region. Accurate pressure prediction in the edge areas of the contact length is important, though, for the simulation of slip development as the corresponding zones are initially generated close to the contact ends [31].

The increase in the modal range of the solution leads to the transition from parabolic to trapezoidal distribution shape in the low deformation case (fig. 20(a)). A frequency range of $1000 \mathrm{~Hz}$ is capable of predicting less than $5 \%$ deviation along the whole length, although the frequency required for capturing the concave shape at the centre is much higher $(1401 \mathrm{~Hz})$.

The shape effect of the solution frequency range in the high deformation case is far more complicated (fig.20(b)). It is evident that (excluding the areas very close to the edges of the contact) the same reduction level results in smaller deviation from the complete solution, in comparison to the $d=10 \mathrm{~mm}$ case. The requirement of $5 \%$ maximum deviation is fulfilled by a $600 \mathrm{~Hz}$ solution range. The reason for the better correlation can be identified in the relative participation factors of the modes (fig. 10(a)).

The effect of modal reduction on the prediction of the horizontal pressure distribution is presented in fig. 21. Although the deviation near both the edge and the axis of symmetry of the contact seems to be huge, it should be noted that the actual corresponding values are insignificant. The shrinkage of the modal range results in lower pressure values near the the contact end but higher ones close to the centre, in the low deformation case. A more complicated shape effect is identified in the deviation of the high deformation case.

\section{CONCLUSIONS}

A two dimensional transient tyre model has been developed and used for the simulation of the tyre contact behaviour. The core of the model consists of the established ring on elastic foundation model, subject to a modal expansion/reduction process. The model incorporates a sidewall structure with radial non linearity and a discretized tread viscoelastic foundation able to predict local slip/adhesional behaviour.

The contact simulation potential of the model is qualitatively validated with published experimental studies. The non linear contact behaviour is investigated as a function of the imposed vertical load/deformation. The interaction between the carcass deformation pattern and magnitude with the predicted vertical and horizontal contact stress field is identified. The parabolic distribution shape is transformed into trapezoidal and a central concave section is generated for increasing load. Accordingly, a radially inwardsconcave-deformation section is created in the central contact part of the belt.

Since the carcass deformation is simulated through the modal expansion technique, the contact behaviour is for the first time associated with certain modes participation in the resulting deformation response. Increasing deformation, although resulting in higher participation of the first modes, narrows the range of the relative modal participation. As a result, the modal range required for achieving a certain level of accuracy in the prediction of the contact pressure shrinks with the imposed load.

The central concave formation is associated with the 
participation of certain modes, the modal number of which depends on the extent of the effect. Cases of low loads are associated with high modal number modes, while increasing load results in the participation of lower modes.

The above results offer a satisfactory explanation of the modal reduction effect across a deformation range, not only in terms of total reaction force, but also in terms of stress distribution along the contact length. As expected, extended modal reduction results in stiffer contact behaviour, which might counteract the sidewall buckling/softening effect. Although for extensive modal reduction the vertical force overestimation is more intense in the high deformation case, the computational error of the low deformation case remains more significant as the level of modal reduction increases.

The broader modal range based solution captures the transformation of the predicted vertical distribution shape from parabolic to trapezoidal in the low deformation case, a result that can be qualitatively predicted through the observation of the associated mode shapes. The shape effect, though, in the high deformation case cannot be easily identified, as the level of prediction of the concave formation affects the observed deviation along the contact length. In accordance to the relative modal participation conclusions, the same reduction level leads to larger relative deviation values in the low load application. In any case, the effect of the reduction is more evident near the edges of the contact, significantly affecting the local slip prediction potential of the model. Additionally, a far broader modal range is required for the accurate prediction of the pressure distribution, while the calculation of the total vertical load appears to be less demanding in this respect.

Imposing a certain cut-off frequency on the reduction process bears a stronger computational error in the prediction of the horizontal pressure field. As the tangential belt modes, which mainly affect the horizontal contact deformation, appear higher than the corresponding radial ones in the general frequency order, the frequency based reduction mainly cuts out tangential modes. Underestimation near the edges and overestimation near the centre is the obvious reduction effect in the low load case. No similar conclusion can be drawn in the high deformation case, apart from the prediction of lower deviation values.

The above results may offer valuable directions for the application of modal expansion/reduction techniques in many two-dimensional modelling approaches, as the derived qualitative conclusions are based on the actual physical mechanism involved and are not ex- clusively associated with the ring model here exploited. The conclusions on the complicated relationship between the frequency/modal range of solution and the computational error can be applied for the significant reduction of the solution time in various tyre simulation studies.

\section{REFERENCES}

[1] T. Akasaka, M. Katoh, S. Nihei, and M. Hiraiwa, "Twodimensional contact pressure distribution of a radial tire," Tire Science and Technology, vol. 18, no. 2, pp. 80-103, 1990.

[2] H. Shiobara, T. Akasaka, S. Kagami, and S. Tsutsumi, "One-dimensional contact pressure distribution of radial tires in motion," Tire Science and Technology, vol. 23, no. 2, pp. $116-135,1995$.

[3] P. Sujin, Y. Wansuk, C. Jinrae, and K. Beomsoo, "Pressure-sensing pad test and computer simulation for the pressure distribution on the contact patch of a tyre," Proceedings of the Institution of Mechanical Engineers, Part D: Journal of Automobile Engineering, vol. 221 , no. 1 , pp. $25-31,2007$.

[4] E. Sakai, "Measurement and visualization of the contact pressure distribution of rubber disks and tires," Tire Science and Technology, vol. 23, no. 4, pp. $238-255$, 1995.

[5] M. Pau, B. Leban, and A. Baldi, "Ultrasonic measurements of contact area and pressure distribution of a pneumatic tire on a rigid surface," Tire Science and Technology, vol. 36, no. 1, pp. $43-62,2008$.

[6] J. Pelc, "Towards realistic simulation of deformations and stresses in pneumatic tyres," Applied Mathematical Modelling, vol. 31, no. 3, pp. 530-540, 2007.

[7] X. Zhang, S. Rakheja, and R. Ganesan, "Estimation of tyre-road contact pressure distribution based on nonlinear finite element analysis," Heavy Vehicle Systems, vol. 8, no. 3-4, pp. $197-217,2001$.

[8] M. Gipser, "FTire: a physically based applicationoriented tyre model for use with detailed MBS and finite-element suspension models," Vehicle System Dynamics, vol. 43, no. 1, pp. 76-91, 2005.

[9] T. Akasaka and K. Kabe, "Deformation and cord tension of a bias tire in contact with the road," Tire Science and Technology, vol. 5, no. 4, pp. 171 - 201, 1977.

[10] K. Kabe and T. Akasaka, "Deformation and cord tension of a radial ture in contact with the road," American Society of Mechanical Engineers, Applied Mechanics Division, AMD, vol. 40, pp. 41 - 56, 1980.

[11] F. Böhm, "Mechanik des gürtelreifens," IngenieurArchiv, vol. 35, no. 2, pp. 82-101, 1966.

[12] Y. Chang, T. Yang, and W. Soedel, "Dynamic analysis of a radial tire by finite elements and modal expansion," Journal of Sound and Vibration, vol. 96, no. 1, pp. 111, 1984.

[13] P. Zegelaar, "Modal analysis of tire in-plane vibrations, SAE paper 971101," SAE International Congress and Exposition, Detroit, USA, February, pp. 24-27, 1997. 
[14] J. Perisse, J. Clairet, and J. Hamet, "Modal testing of a smooth tire in low and medium frequency- estimation of structural parameters," SPIE proceedings series, pp. 960-967, 2000.

[15] W. Soedel, Vibrations of Shells and Plates. CRC Press, 2004.

[16] S. Kim and A. Savkoor, "The contact problem of inplane rolling of tires on a flat road," Vehicle System Dynamics, vol. 27, pp. 189-206, 1996.

[17] P. Zegelaar, S. Gong, and H. Pacejka, "Tyre models for the study of in-plane dynamics," Vehicle System Dynamics, vol. 23, pp. 578-590, 1994.

[18] H. Pacejka, Tyre and Vehicle Dynamics. ButterworthHeinemann, 2006.

[19] G. Mavros, H. Rahnejat, and P. King, "Transient analysis of tyre friction generation using a brush model with interconnected viscoelastic bristles," Proceedings of the Institution of Mechanical Engineers, Part K: Journal of Multi-body Dynamics, vol. 219, no. 3, pp. 275283, 2005.

[20] S. Kim, P. E. Nikravesh, and G. Gim, "A twodimensional tire model on uneven roads for vehicle dynamic simulation," Vehicle System Dynamics, vol. 46, no. 10 , pp. $913-930,2008$.

[21] W. Soedel and M. Prasad, "Calculation of natural frequencies and modes of tires in road contact by utilizing Eigenvalues of the axisymmetric non-contacting tire," Journal of Sound and Vibration, vol. 70, no. 4, pp. 573$584,1980$.

[22] S. Huang, "Vibration of rolling tyres in ground contact," International Journal of Vehicle Design, vol. 13, no. 1, pp. 78-95, 1992.

[23] P. Zegelaar and H. Pacejka, "The in-plane dynamics of tyres on uneven roads," Vehicle System Dynamics, vol. 25, pp. 714-730, 1996.

[24] F. Wullens and W. Kropp, "A Three-Dimensional Contact Model for Tyre/Road Interaction in Rolling Conditions," Acta Acustica united with Acustica, vol. 90, no. 4, pp. 702-711, 2004.

[25] C. Mousseau and G. Hulbert, "The dynamic response of spindle forces produced by a tire impacting large obstacles in a plane," Journal of Sound and Vibration, vol. 195, no. 5, pp. 775-796, 1996.

[26] A. Miège and A. Popov, "Truck tyre modelling for rolling resistance calculations under a dynamic vertical load," Proceedings of the Institution of Mechanical Engineers, Part D: Journal of Automobile Engineering, vol. 219, no. 4, pp. 441-456, 2005.

[27] F. Bowden and D. Tabor, The Friction and Lubrication of Solids. Oxford University Press, 2001.

[28] S. Clark, "Mechanics of Pneumatic Tires, Monograph no.122," National Bureau of Standards Washington DC, 1981.

[29] W. Hall, J. Mottram, D. Dennehy, and R. Jones, "Characterisation of the contact patch behaviour of an automobile tyre by physical testing," International Journal of Vehicle Design, vol. 31, no. 3, pp. 354 - 376, 2003.
[30] A. Savkoor, "Some aspects of friction and wear of tyres arising from deformations, slip and stresses at the ground contact," Wear, vol. 9, pp. 66-78, 1966.

[31] J. Maurice and A. Savkoor, "Influence of flexibility properties and friction laws on tyre behaviour," Vehicle System Dynamics, vol. 37, no. suppl., pp. 107-124, 2002.

\section{APPENDIX}

MODEL PHYSICAL PROPERTIES

\begin{tabular}{lllr}
\multicolumn{4}{c}{ Ring properties } \\
\hline elastic modulus & $E$ & {$\left[\frac{\mathrm{kgr}}{\mathrm{m} \cdot \mathrm{sec}}\right]$} & $10.4 \times 10^{9}$ \\
density & $\rho$ & {$\left[\frac{\mathrm{kg} r}{\mathrm{~m}^{3}}\right]$} & $8.1 \times 10^{9}$ \\
radius & $r$ & {$[\mathrm{~m}]$} & 0.3 \\
section height & $d$ & {$[\mathrm{~m}]$} & $3.1 \times 10^{-3}$ \\
section width & $b$ & {$[\mathrm{~m}]$} & $15.2 \times 10^{-2}$ \\
viscous factor & $\lambda$ & {$\left[\frac{\mathrm{kgr}}{\mathrm{m}^{2}}\right]$} & $0.1 \times 10^{5}$ \\
inflation pressure & $P_{0}$ & {$[\mathrm{~Pa}]$} & $2.2 \times 10^{5}$ \\
\hline \hline
\end{tabular}

Sidewall properties

\begin{tabular}{lllr}
\hline radial stiffness $^{\mathrm{a}}$ & $k_{r}$ & {$\left[\frac{\mathrm{kgr}}{\mathrm{m}_{\mathrm{m} . \mathrm{sec}^{2}}}\right]$} & $192.9 \times 10^{6}$ \\
tangential stiffness $^{\mathrm{a}}$ & $k_{t}$ & {$[\mathrm{mgr}$} \\
string length $^{\mathrm{b}}$ & $l_{\text {string }}$ & {$[\mathrm{m}]$} & $648.7 \times 10^{5}$ \\
wheel radius & $R_{w}$ & {$[\mathrm{~m}]$} & $12.2 \times 10^{-2}$ \\
\hline \hline
\end{tabular}

\begin{tabular}{lllr}
\multicolumn{4}{c}{ Tread properties } \\
\hline radial stiffness $^{\mathrm{c}}$ & $K_{r}$ & {$\left[\frac{\mathrm{kgr}}{\mathrm{sec} \mathrm{c}^{2}}\right]$} & $30.2 \times 10^{4}$ \\
radial damping coef. & $C_{r}$ & {$\left[\frac{\mathrm{kgr}}{\mathrm{sec}}\right]$} & 755 \\
tangential stiffness & $K_{t}$ & {$\left[\frac{\mathrm{kgr}}{\mathrm{sec}}\right]$} & $10.1 \times 10^{4}$ \\
tangential damping coef. & $C_{t}$ & {$\left[\frac{\mathrm{kgr}}{\mathrm{sec}}\right]$} & 253 \\
radial length & $l_{\text {tread }}$ & {$[\mathrm{m}]$} & $10 \times 10^{-3}$ \\
\hline \hline
\end{tabular}

a after [13]

$\mathrm{b}$ non linear case

c after [23] 